\title{
Measurement of the mean transit time of dietary residue through the human gut
}

\author{
J. H. CUMMINGS, ${ }^{1}$ D. J. A. JENKINS, AND H. S. WIGGINS \\ From the Medical Research Council Gastroenterology Unit, Central Middlesex Hospital, London
}

SUMMARY A new method is described for measuring the mean transit time (MTT) of digesta through the human gut in which a constant amount of marker (radio-opaque pellets) is fed to subjects with each meal over a period of weeks, and its excretion measured in the stools. The MTT measured by this method (MTT-C) has been compared with MTT measured by giving single doses of similar markers to the same subjects (MTT-S) and with the $80 \%$ transit time (80\% TT). Mean values on three dietary regimes for the MTT-C $(54 \cdot 2 \mathrm{~h} \pm 2 \cdot 5)$ and MTT-S $(54 \cdot 2 \mathrm{~h} \pm 2 \cdot 6)$ were lower than that for $80 \%$ TT $(63.1 \mathrm{~h} \pm 3.0)$. The average MTT-C in a group of six healthy young men on an ad libitum diet was $2 \cdot 3$ days (range $0 \cdot 7-4 \cdot 0$ ). When additional dietary fibre was added to a standard diet the average MTT-C fell (in all of five subjects) from 2.4 to 1.6 days. A continuous record of MTT-C is obtained by this new method which shows wide variations from week to week even on controlled dietary intakes. Using the single dose technique, evidence is produced which suggests that the MTT-S is a more accurate and reproducible method than the $80 \%$ TT.

The length of time that dietary residue remains in the colon is important in determining colonic metabolism. It is thought to be one of the factors which affects the occurrence of large bowel cancer (Burkitt, 1971) and may be relevant to the extent to which the gut microflora metabolize foreign organic compounds (Scheline, 1973) and dietary fibre (Southgate, 1973). It is also important in calculating the dose of radiation received from ingested radioactive material (Eve, 1966).

A number of different techniques have been used to measure the time that food residue takes to pass through the bowel, all of which are based on the same principle. This is to give a single dose of marker and measure its appearance in the stools. Few investigators have considered the effect on the interpretation of their data of the way in which residue passes through the gut, particularly the effect of mixing (Alvarez, 1948; Cassano et al., 1968; Edwards and Beck, 1971; Findlay et al., 1974) and of day to day variations in bowel habit. Only in the work of Davignon et al. (1968) has a method using a different principle been used.

'Present address: Dunn Nutrition Laboratory, Milton Road, Cambridge.

Received for publication 4 December 1975
We have measured the mean transit time (MTT) of dietary residue through the gut by giving a constant number of radio-opaque pellets each day to healthy volunteers continuously over a number of weeks (MTT-C) and also by giving single doses of pellets (MTT-S) and recording the amount excreted daily in the stool. These data have been compared with that of the $80 \%$ transit time $(80 \%$ TT) using the method of Hinton et al. (1969). The efficacy of these methods in detecting changes in transit due to diets high or low in dietary fibre has been observed.

\section{Methods}

\section{SUBJECTS}

Twelve healthy male subjects aged from 22-32 years who were either medical students or members of staff took part in 23 studies. The protocol was fully explained to each subject and had been approved by the ethical committee of the Central Middlesex Hospital.

\section{MARKERS}

For the continuous marker studies the marker used was radio-opaque barium sulphate impregnated polyethylene pellets (Portex Ltd.) which were approximately cube shaped, specific gravity 1.25 , and mean weight $31 \mathrm{mg}$. They were treated with a 
blast of hot air from a hair dryer to round off the sharp corners and seived through a coarse wire mesh to exclude uneven pellets. Three other types of marker were made for use in the single dose studies. The large pellets were cut into four smaller pellets with a mean weight of $7.8 \mathrm{mg}$ and the same specific gravity of $1 \cdot 25$. Additional markers were obtained by cutting $1 \cdot 1 \mathrm{~mm}$ sections from radio-opaque PVC tubing of $3.0 \mathrm{~mm}$ external diameter (Portex R4) and $1.1 \mathrm{~mm}$ sections from similar tubing of $4.5 \mathrm{~mm}$ external diameter (Portex R5). These had a specific gravity of 1.63 , mean weight of 8 and $16 \mathrm{mg}$ respectively, and were called small and large circles. These four different markers were clearly distinguishable by their shape in the stools on radiography (Fig. 1).

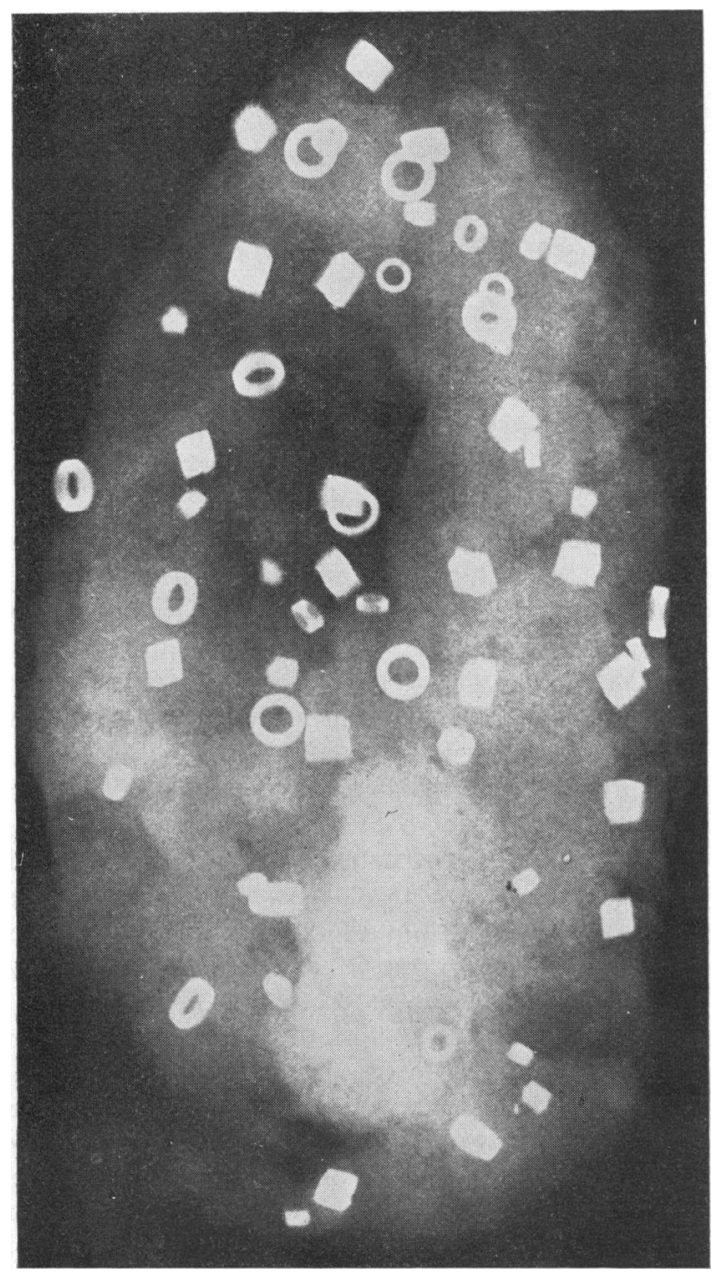

Fig. 1 Radiograph of a stool containing all four types of marker. These are evenly distributed and clearly distinguishable in the stool.
PLAN OF STUDY

Four experimental plans were followed:

1. Continuous marker-ad libitum diets

Six subjects (studies 1-6) were studied for four weeks while eating their normal unrestricted diet.

\section{Continuous marker-controlled diets}

Five subjects (studies 7-11) undertook a six week controlled metabolic diet experiment in which they ate a carefully prepared diet while carrying out their normal occupations and living in a hostel in the hospital grounds. The basic diet was prepared in the metabolic kitchen and was of a normal 'Western' type composition, low in dietary fibre, comprising $2802 \mathrm{Kcal}, 351 \mathrm{~g}$ carbohydrate, $122 \mathrm{~g}$ fat, $88 \mathrm{~g}$ protein, and $3.6 \mathrm{~g}$ crude fibre. Three one day menus of this composition were designed and fed in rotation for three weeks. For another three week period the fibre content of the diet was changed by substituting an equal weight of wholemeal bread for white bread (120 g), All-bran for cornflakes (25 g), bran biscuits for Nice biscuits ( $52 \mathrm{~g}$ ), and by adding $30 \mathrm{~g}$ of bran (Allinson's Bran Plus). The composition of the high fibre diet was $2755 \mathrm{Kcal}, 339 \mathrm{~g}$ carbohydrate, $115 \mathrm{~g}$ fat, $97 \mathrm{~g}$ protein, and $11.7 \mathrm{~g}$ crude fibre. While data were not available for the dietary fibre content (as opposed to crude fibre content) of all the dietary items, it was available for the dietary fibre content of the four items changed in the two diets using the methods of Southgate (1969). From this it was calculated that the high fibre diet contained an additional $36 \mathrm{~g}$ of dietary fibre.

In studies 7-9 the standard diet was taken first and in studies 10-11 the high fibre diet first. Full details of the diets are available in Jenkins et al. (1975).

In two further studies (12-13) two additional subjects were studied for a continuous period of six weeks in which they took only the basic diet.

In all these studies (1-13) the subjects took five pellets with each meal (15 per day) throughout the experimental periods. During this time and for one week after the markers had been discontinued the subjects collected their faeces. Each stool was collected separately in a plastic bag fixed over a toilet. The bag was then sealed, labelled, and immediately cooled to $-20^{\circ} \mathrm{C}$. The number of markers excreted in the stool was found by $x$-raying every stool passed. Two films (3M Type R, no screen) were taken of each stool at $300 \mathrm{~mA}$ and $60 \mathrm{kV}$ for $0.4 \mathrm{~s}$ at a distance of $50 \mathrm{~cm}$ from the tube. The specimen was rotated through $90^{\circ}$ between exposures.

\section{Single dose-20 markers}

Twenty markers, either small pellets, large, or small 
circles, were given to the subjects during studies 1-13 on 50 occasions, after marker equilibrium for the continuous marker had been reached.

\section{Single dose -80 markers}

In order to detect any possible differences in the way the four markers behaved while passing through the gut a single dose of 80 markers containing 20 of each type was given with breakfast on 10 occasions to eight subjects (not participating in the continuous marker studies at the time) and the stools collected until all the markers had been recovered.

TERMS AND CALCULATIONS

Mean transit time (MTT) This is the average time that marker takes to pass through a system.

MTT-S Where the marker is given as a single dose the MTT is calculated as follows:

$$
\operatorname{MTT}-S(h)=\sum_{\substack{i=1 \\ i=n}}^{i=n} x_{i} t_{i}
$$

where $x_{1}$ is the number of radio-opaque pellets present in the stool passed after time interval $t_{i}$.

MTT-C Where the marker is given continuously to the subject the MTT is calculated as the turnover time, which is the same as the MTT (Zilversmit, 1960).

$$
\begin{aligned}
\text { MTT-C }(\text { days })= & \text { Turnover time }= \\
& \frac{\text { Mean marker pool size }}{\text { Number of markers ingested daily }}
\end{aligned}
$$

Mean marker pool (MMP) This is the sum of the products of the number of markers $\left(\mathrm{x}_{\mathrm{i}}\right)$ and the period of time $\left(t_{i}\right)$ during which that number of markers are present in the gut. The total number of periods is determined by the frequency with which the number of markers present changes either by ingestion or defaecation and includes the final period from the last change to midnight. It is the average number of markers present in the gut during the day in question.

$$
\operatorname{MMP}=\frac{\sum_{i=1}^{i=n} x_{i} t_{i}}{\sum_{i=1}^{i=n} t_{i}}
$$

The denominator should equal 24 .

Eighty per cent transit time $(80 \%$ TT) This is the time from ingestion of a dose of marker to the appearance of a stool that, together with any previous stools, contains $80 \%$ of the markers ingested (Hinton et al., 1969).

Five day moving average In order to reduce the day to day variations in marker excretion and MMP due to the infrequent and variable nature of faecal output while preserving the advantage of daily measurements the results have been expressed as five day moving averages-that is, mean of days 1-5, 2-6, 3-7, etc. These have been plotted in Figs. 2-4 at the fifth day of each period.

Equilibrium Marker equilibrium is attained when the mean daily output of marker is equal to the marker input. Because of the inherently wide variations in daily marker output, we have taken equilibrium to be present when the mean daily marker output varied by not more than \pm one pellet $(7 \%)$ from the ideal state during three consecutive five day moving average periods-that is, seven days. Equilibrium is analogous to a steady state as used in the kinetic analysis of metabolic studies (Zilversmit, 1960) but the use of the term 'steady state' implies a constancy of the rate of output that is not justified in data referable to faeces.

\section{Results}

\section{MARKER RECOVERY}

Of the 6730 pellets given in the continuous marker studies (1-13), 6710 were recovered. Except for two studies $(2,7)$ where the final stool collected at the end of the last week still contained markers, marker recovery was always within two pellets of being complete. Recovery in study 2 was $416 / 420$ and in study 7, 622/630.

Calculation of the marker pool based on the difference between total marker input and total output is subject to a cumulative error of the daily measurements of marker input and output. This error has been calculated for each study by expressing the actual number of markers recovered after midnight on the final study day as a percentage of the calculated number of markers retained (total marker input - total marker output) at midnight on that day. The mean recovery was $97.6 \% \pm 3.0 \%$ giving a mean error in the estimation of the final marker pool of $2.4 \%$ with the maximum error, study 4 , being $7 \cdot 7 \%$. Although there are several possible sources of error in estimating the number of markers retained-namely, failure of the subject to take the marker, an error in measuring the dose, loss of stools, poor visualization on radiography, particularly if fluoroscopy is used instead of $x$-ray film, these results show that the errors are small if due care is taken. 


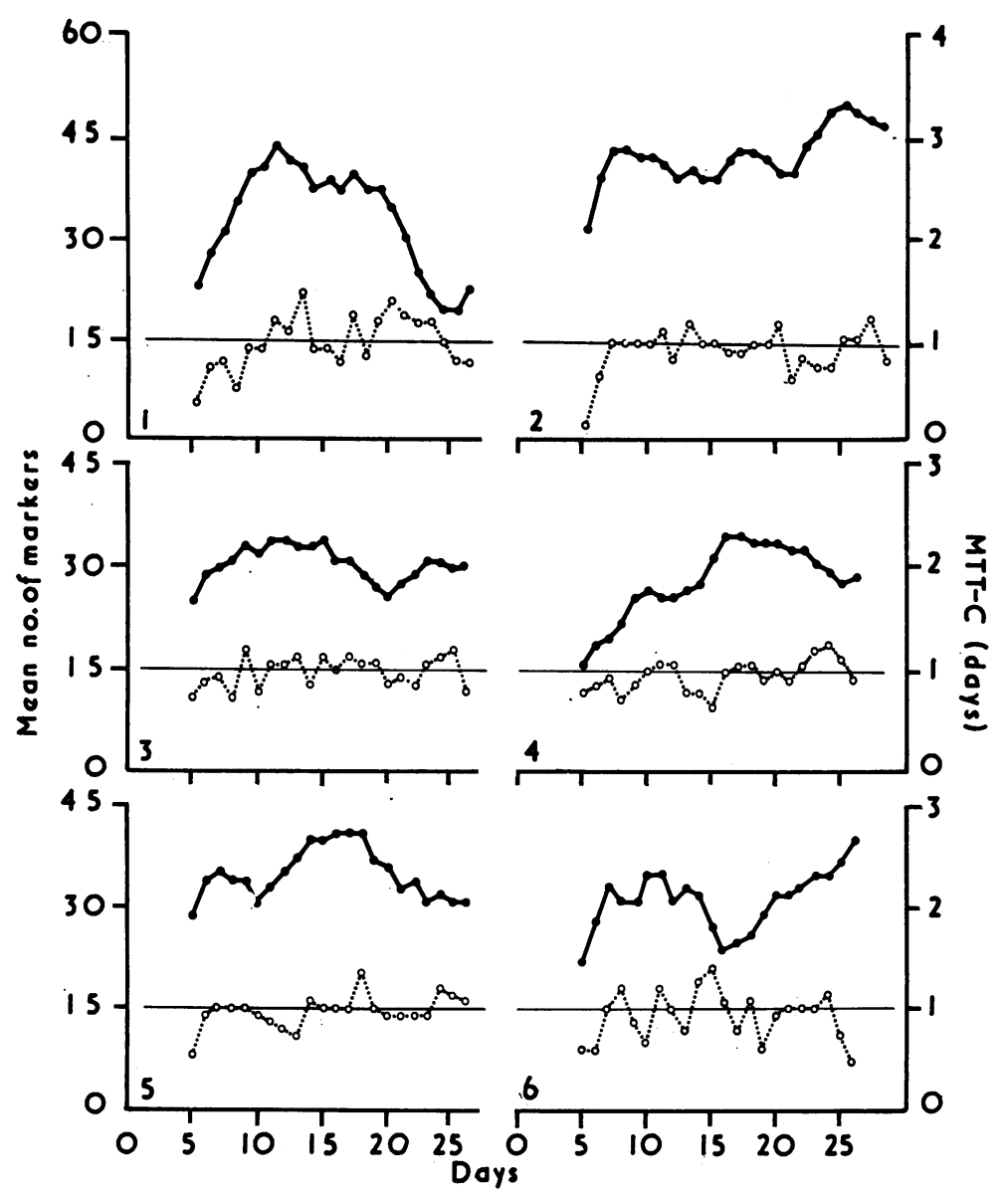

Fig. 2 Five-day moving average of mean marker pool, MTT-C, and faecal marker output for four week periods on ad libitum diets.

= mean marker pool size $\bigcirc--\bigcirc=$ faecal marker output

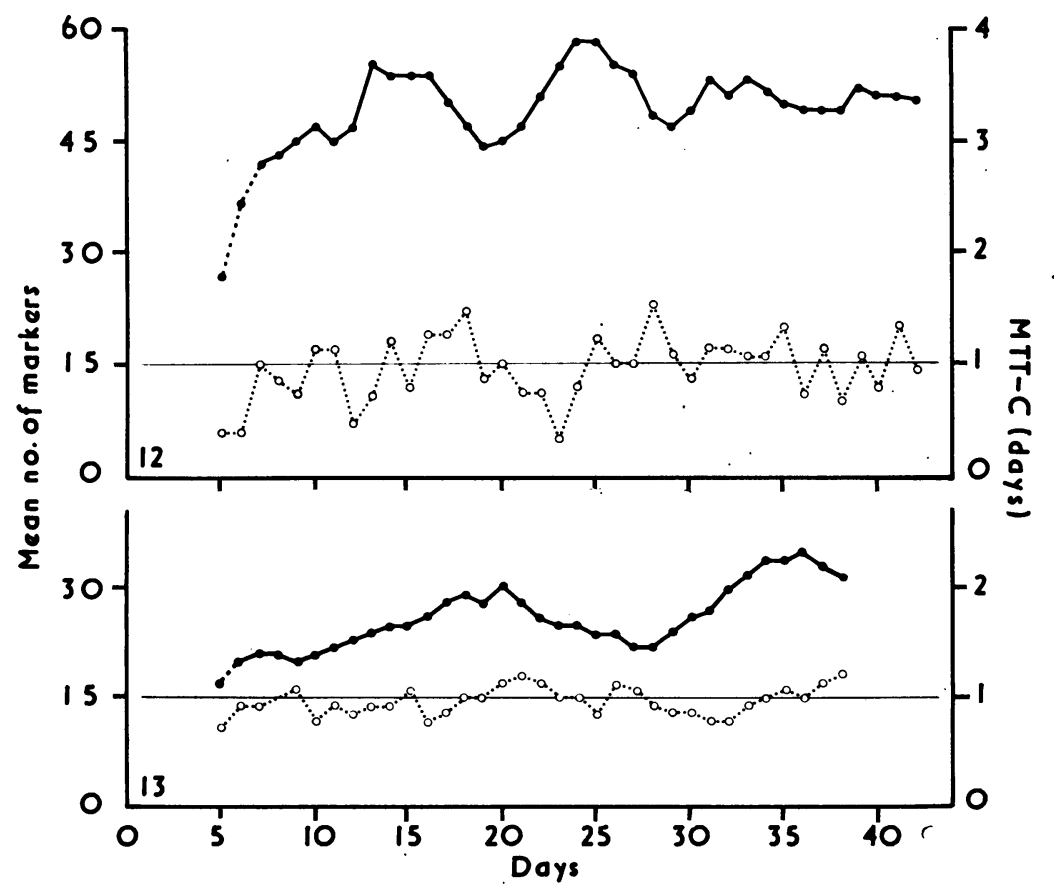

Fig. 3 Five-day average of mean marker pool, MTT-C, and faecal marker output in six week studies on standard diet.

(Other symbols as in Fig. 2.) 


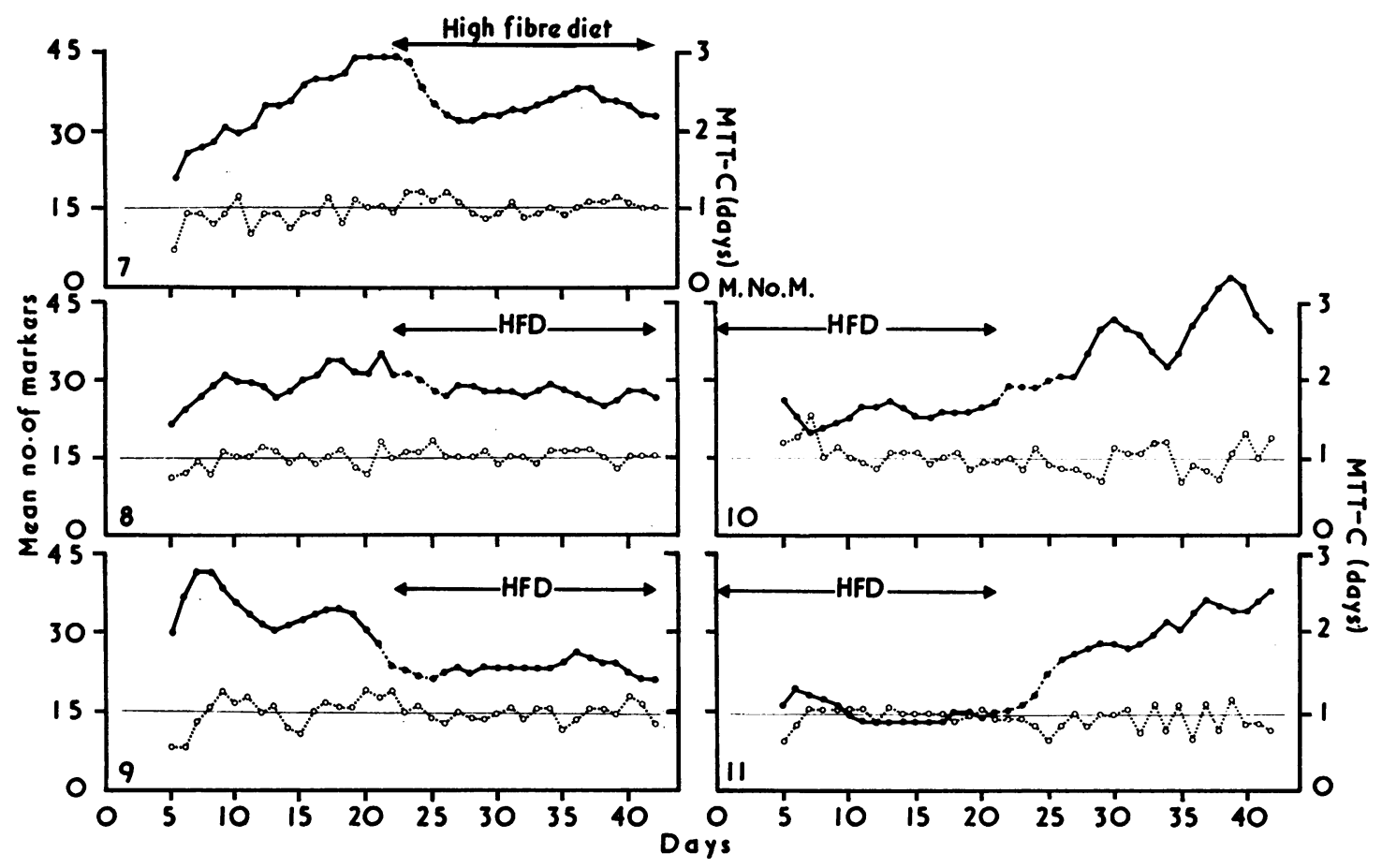

Fig. 4 Five-day moving average of mean marker pool, MTT-C, and faecal marker output during three-week standard diet and three week high fibre diet (HFD) periods. - - - $=$ first four days of new diet .

In these studies four different markers were used which ranged in weight from 7.8 to $31.0 \mathrm{mg}$, in specific gravity from 1.25 to 1.63 , and in shape. As specific gravity is thought to influence the way in which unabsorbed material passes through the gut (Hoelzel, 1930; Kirwan and Smith, 1974), all four markers were given together on 10 occasions to eight subjects and the MTT-S calculated for each. The mean, SEM, and range in the 10 studies for each marker was: large circles $57.0 \pm 6.1$ range 37-86 $\mathrm{h}$, large pellets $53.0 \pm 7.4$ range $23-89 \mathrm{~h}$, small pellets $54.9 \pm 6.6$ range $25-80 \mathrm{~h}$, and small circles $57.4 \pm 6.8$ range $37-89 \mathrm{~h}$. The difference between the markers was not significant when tested by analysis of variance $(F=0.09)$ or when tested by paired $t$ test (six comparisons, $P$ in all cases $>0.05$ ), and therefore the markers have been considered to be interchangeable for the purpose of this investigation.

No unwanted effect attributable to the markers was observed in any of the subjects, who took them often in large numbers without difficulty.

CONTINUOUS MARKER STUDIES (MTT-C) Figures 2-4 show the mean marker pool size, faecal marker output, and MTT-C for each study expressed as five day moving averages. When this method of marker administration is used, a continuous record of MTT-C is obtained. In the ad libitum diet studies (Fig. 2, 1-6), it may be seen that there are wide variations from week to week in MTT-C and few occasions when faecal marker output equals input for any length of time. This week to week variation is seen even when subjects are kept on a constant diet (Fig. 3) for up to six weeks. On the high fibre diet, however (Fig. 4), equilibrium (by our definition) exists for quite long periods of time and MTT-C is fairly constant.

Because of these fluctuations in MTT-C, a single figure for an individual can be obtained only by averaging the daily MTT-C values. In these studies this has been done by averaging the MTT-C values from all the days after a period of three times the maximum MTT-C value found (to allow for equilibration of marker).

The average MTT-C in studies 1-6 (ad libitum diets) is $2 \cdot 3$ days with a range of $0 \cdot 7-4 \cdot 0$ days. This is not significantly different from the MTT-C in the standard diet period of studies 7-11 but during the high fibre diet periods in these studies MTT-C falls 
significantly from 2.4 to 1.6 days $\left(t_{4}=3.44, \mathrm{P}<\right.$ $0.05)$. In all five studies (7-11) the addition of fibre to the diet shortened MTT-C.

COMPARISON OF MTT-C WITH SINGLE DOSE METHODS (MTT-S AND $80 \%$ TT)

A comparison between the continuous marker (MTT-C) and single dose methods (MTT-S and $80 \%$ TT) of measuring transit is shown in Fig. 5. The mean value for MTT-C obtained for the period during which the single dose markers were excreted is used for the comparison (50 comparisons in studies 1-13). For each single dose of marker both MTT-C and $80 \%$ TT has been calculated. Both single dose methods correlate quite well with MTT-C, although that of MTT-S is slightly better $(\mathrm{R}=0.87)$ than that for $80 \%$ TT $(\mathrm{R}=0.78)$. Inspection of Fig. 5 confirms that the scatter of values using the $80 \%$ TT is noticeably greater than for MTT-S.

The greater precision of the MTT-S when compared with the $80 \%$ TT is also clearly shown by the 10 studies in which all four markers were given together as a single dose. For each study, the MTT-S and $80 \%$ TT has been calculated for each marker. Each individual MTT-S or $80 \%$ TT has then been expressed as a percentage of the average of the four MTT-Ss or four $80 \%$ TTs for that study and the coefficient of variation calculated. For all 10 studies the coefficient of variation for the MTT-S is $9.5 \%$ and for $80 \%$ TT $23.7 \%$.

The greater accuracy of the MTT-S rather than the $80 \%$ TT analysis of a single dose of marker may also be found from the mean and SEM of all 50 values for each method. MTT-C 54.2 $\pm 2.52 \mathrm{~h}$; MTT-S $54.2 \pm 2.59 \mathrm{~h} ; 80 \%$ TT $63.1 \pm 3.0 \mathrm{~h}$. The difference between MTT-C and $80 \%$ TT is significant, $t=2.231 \mathrm{P}<0.05(\mathrm{df}=48)$. The MTT-C therefore gives a lower value for transit than does the $80 \%$ TT but one which is very close to the MTT-S.

\section{Discussion}

REQUIREMENTS OF A NEW METHOD FOR MEASURING TRANSIT

A variety of methods have been used to estimate the time taken for food residue to pass through the gut. All of these, however, are based on the same principle, that of giving a single dose of marker to a subject and recording its appearance in the stools. Using this single dose technique the markers used have included bismuth (Hertz et al., 1907), coloured dyes (Mulinos, 1935), barium (Wallace et al., 1938), glass beads (Alvarez and Freedlander, 1924), various seeds, gravel, steel ball bearings and silver wire (Hoelzel, 1930), millet (Burnett, 1923), radioactive chromic oxide and sodium chromate (Hansky and Connell, 1962), and the radio pill (Connell and Rowlands, 1960). A review of the data in these studies shows a wide range of values for normal people of one to five days with the majority passing the marker in three days. Except in the study of Davignon et al. (1968), none of these methods has

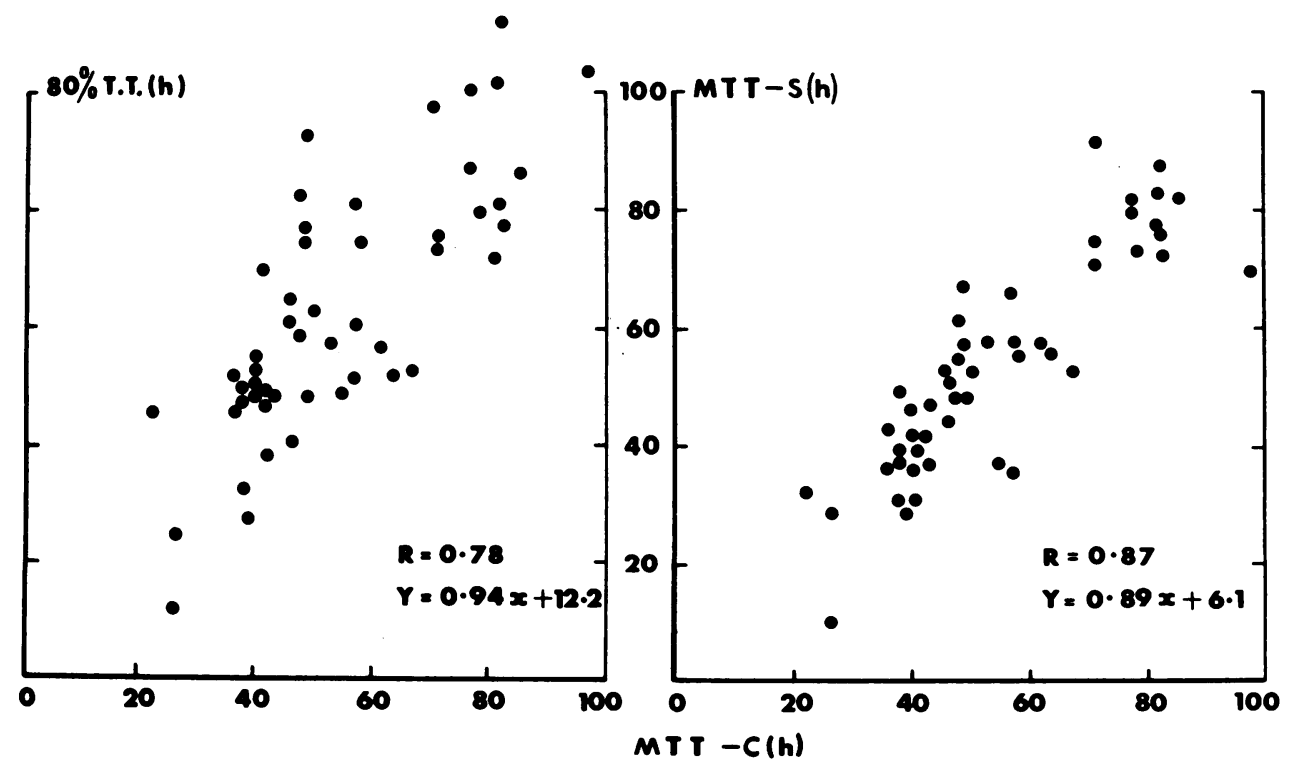

Fig. 5 Comparison of MTT-C and $80 \%$ TT with MTT-C (50 comparisons in studies 1-13). 
previously been tested against one requiring a different principle for the measurement of transit. All of them suffer in the way that they are analysed from the theoretical disadvantage that they take no account of the apparently exponential decay that occurs in the recovery of a single dose of marker from the gut. They are also vulnerable to short term, day to day variations in bowel habit.

Furthermore, when used to detect differences in transit due to dietary changes, conflicting results have been recorded even when identical methods are used. Using the $80 \%$ TT Payler et al. (1975) suggest that fibre slows transit in persons where it is rapid-that is, one day or less and shortens it where transit time is long, while Eastwood et al. (1973) were unable to demonstrate a significant change in normal subjects when $16 \mathrm{~g}$ of cellulose or bran was added to the diet.

In view of these problems, an alternative method is required which makes no assumptions about the kinetics of the system, is not subject to day to day variations in bowel habit, is simple to perform, practical, and accurate. The continuous administration of radio-opaque pellets as a marker satisfies these criteria.

SUITABILITY OF RADIO-OPAQUE PELLETS AS A MARKER

The radio-opaque markers used in this study conform with a number of 'ideal' characteristics that are required of markers used to investigate gastrointestinal function. They are unabsorbed, non-toxic, easy and safe to measure, and do not of themselves alter gut metabolism. Their main value, however, depends on how closely they mimic the natural dietary residue. Dietary residue is made up of a large number of substances including dietary fibre, which is itself a complex mixture of plant material (Cummings, 1973). The methods of analysis and labelled compounds necessary for the conduct of a direct comparison with markers of any sort are not available.

Indirect evidence suggests that the markers should behave in a similar manner to dietary residue. One of the factors which is known to affect the passage of particles through the gut is specific gravity (Hoelzel, 1930; Kirwan and Smith, 1974). In general, substances with a high specific gravity such as glass beads $(2 \cdot 6)$ travel faster than those with a lower specific gravity such as knots of cotton $(1 \cdot 45)$. Whole faeces which contain bacteria, epithelial cells, mucus, trapped air, and the remnants of gastrointestinal secretions are about SG 1.0 (Levitt and Duane, 1972), while cellulose is $1 \cdot 3$ to 1.6 (West, 1973-74). The specific gravity of the radio-opaque pellets is in the range $1 \cdot 3-1 \cdot 6$. The failure to demonstrate a difference in the MTT-S for the four different markers used in the present study, when given simultaneously, indicates that within this range of size and specific gravity large differences in behaviour will not be found.

Additional indirect evidence is obtained from observation of radiographs of the stool which show the markers to be evenly distributed throughout the stool (Fig. 1).

\section{COMPARISON OF METHODS}

Measurement of MTT by the continuous marker method provides a value that is characteristic for the system but makes no assumptions about its kinetics. Furthermore, continuous analysis of the data enables a five day mean to be taken, thus overcoming the day to day variations due to changes in bowel habit. When the three methods (MTT-C, MTT-S, and $80 \%$ TT) are compared (Fig. 5), the overall agreement is good. However, the mean value for the MTT-C $(54.2 \mathrm{~h})$ differs significantly from that for $80 \%$ TT $(63 \cdot 1 \mathrm{~h})$. The slower value for $80 \%$ TT is predictable from the way in which the appearance of markers with this type of method is analysed. Similarly, the identical results from MTT-C and MTT-S would be expected on the same grounds. The value obtained by the MTT methods is probably closer to physiological events, as account is taken of the passage of the complete dose of marker, or of each unit of marker in the MTT-C, and not an arbitrary amount such as $80 \%$.

The mean value for the MTT-C in studies 1-6 (ad libitum diets) is 2.3 days, which falls within the range of one to five days found by other workers using a variety of methods. From these data and the indirect evidence suggesting a close identity of the pellets with dietary residue, it can be concluded that the continuous administration of this marker gives a valid measure of MTT and that in this group of six healthy young men a range of $0 \cdot 7-4.0$ days is normal (Table).

When the MTT-C is used, a significant fall in MTT has been demonstrated from 2.4 to 1.6 days when additional fibre is added to the diet. The clear demonstration in all five studies (7-11) that fibre shortens MTT suggests that cereal fibre and its associated substances do affect transit and is additional evidence that the behaviour of these markers reflects that of dietary residue.

\section{FACTORS AFFECTING MTT IN MAN}

The continuous record of MTT-C obtained shows that fibre is not the only factor affecting the passage of residue through the gut because, even when subjects are on a metabolically controlled diet with a constant fibre intake, wide variations in MTT-C 


\begin{tabular}{|c|c|c|c|c|c|c|}
\hline \multirow{2}{*}{$\begin{array}{l}\text { Study } \\
\text { no. }\end{array}$} & \multicolumn{2}{|c|}{ Ad libitum diet } & \multicolumn{2}{|c|}{ Standard diet } & \multicolumn{2}{|c|}{ High fibre diet } \\
\hline & Mean & Range & Mean & Range & Mean & Range \\
\hline $\begin{array}{l}1 \\
2 \\
3 \\
4 \\
5 \\
6\end{array}$ & $\begin{array}{l}2 \cdot 1 \\
3 \cdot 1 \\
2 \cdot 1 \\
2 \cdot 1 \\
2 \cdot 2 \\
2 \cdot 4\end{array}$ & $\begin{array}{l}0 \cdot 7-3 \cdot 1 \\
2 \cdot 3-4 \cdot 0 \\
1 \cdot 4-2 \cdot 7 \\
1 \cdot 2-2 \cdot 6 \\
1 \cdot 3-3 \cdot 5 \\
1 \cdot 7-3 \cdot 2\end{array}$ & & & & \\
\hline 7 & & & $2 \cdot 8$ & $1.9-3 \cdot 6$ & $2 \cdot 3$ & $2 \cdot 0-2 \cdot 7$ \\
\hline 8 & & & $2 \cdot 1$ & $1 \cdot 3-2 \cdot 6$ & $1 \cdot 8$ & $1 \cdot 5-2 \cdot 3$ \\
\hline 9 & & & $2 \cdot 1$ & $1 \cdot 2-2 \cdot 6$ & 1.6 & $1 \cdot 3-2 \cdot 0$ \\
\hline 10 & & & 2.9 & $2 \cdot 1-3 \cdot 7$ & 1.7 & $1 \cdot 3-2 \cdot 1$ \\
\hline 11 & & & $2 \cdot 5$ & $1 \cdot 9-3 \cdot 3$ & 1.0 & $0.7-1.6$ \\
\hline 12 & & & $3 \cdot 5$ & $2 \cdot 5-4 \cdot 8$ & & \\
\hline 13 & & & 1.8 & $1 \cdot 2-2 \cdot 6$ & & \\
\hline Mean & \multicolumn{2}{|c|}{$2.3 \pm 0.06 \mathrm{SEM}$} & \multicolumn{2}{|c|}{$2.4^{*} \pm 0.17 \mathrm{SEM}$} & \multicolumn{2}{|c|}{$1.6 \pm 0.05 \mathrm{SEN}$} \\
\hline
\end{tabular}

Table MTT-C (days) calculated from marker pool size

*Studies 7-11 only

still occur. In studies 7-11 the mean variation was $62 \%$ (average of ranges expressed as a percentage of mean) while on the ad libitum diet the variation is $76 \%$. Because the main pool of dietary residue is in the colon, one would expect colonic function to be important in determining MT T. Colonic function is affected by somatic activity (Holdstock et al., 1970), frequency of meals (Holdstock and Misiewicz, 1970), sleep (J. J. Misiewicz, personal communication), emotion (Barclay, 1933; Chaudhary and Truelove, 1961), and probably by hormones, although few have been looked at (Connell and Logan, 1967). All of these things are likely to affect transit in addition to diet.

\section{SINGLE DOSE TRANSIT METHODS}

If transit is to be measured using a single dose technique, then the MTT-S method is preferable to the $80 \%$ TT. The MTT-S correlates more closely with the MTT-C $(R=0.87)$ than does the $80 \%$ TT $(R=0.78)$ and gives a value for transit which is probably more physiological. In addition, the better reproducibility of the MTT-S is reflected in the coefficient of variation for the two methods in studies done simultaneously with different markers, this being $9.5 \%$ for MTT-S and $23.7 \%$ for $80 \%$ TT.

CHOICE OF METHOD FOR MEASURING TRANSIT The continuous marker method provides a valid alternative to single dose methods for measuring transit and, in addition, provides more information about transit in individuals. While rather tedious, it is suitable for examining the effect on transit of diet, drugs, and other variables in small groups of individuals. Because of the wide variation in transit in individuals over a period of weeks, single dose methods are likely to prove misleading in these circumstances. However, when larger groups are to be studied, the single dose method (MTT-S) gives a true mean for the group. Unfortunately, all these methods require the collection of faeces over a period of five to seven days which renders them unsuitable for work on an epidemiological scale.

The authors wish to thank Sir Francis Avery Jones, Dr E. N. Rowlands, and Dr T. Smith for helpful advice and criticism. Mrs Penelope Stuart has provided invaluable administrative and secretarial help. Statistical advice and help were obtained from Mr R. Dewar, Department of Community Medicine, Central Middlesex Hospital. We are particularly grateful to the medical students who participated in the study, without whom it would have been impossible. J.H.C. was in receipt of a grant from the British Nutrition Foundation.

\section{References}

Alvarez, W. C. (1948). An Introduction to Gastroenterology, pp. 617-633. 4th edn. Heinemann: London.

Alvarez, W. C., and Freedlander, B. L. (1924). The rate of progress of food residues through the bowel. Journal of the American Medical Association, 83, 576-580.

Barclay, A. E. (1933). The Digestive Tract: a Radiological Study of its Anatomy, Physiology, and Pathology, p. 277. Cambridge University Press: Cambridge.

Burkitt, D. P. (1971). Epidemiology of cancer of the colon and rectum. Cancer, 28, 3-13.

Burnett, F. L. (1923). The intestinal rate and form of the faeces. American Journal of Roentgenology, 10, 599-604.

Cassano, C. C., Vecchio, E. Del, and Capurso, L. (1968). I tempi de transito gastrointestinali. Radiologia Pratica, 18, 207-223.

Chaudhary, N. A., and Truelove, S. C. (1961). Human colonic motility: a comparative study of normal subjects, patients with ulcerative colitis and patients with the irritable bowel syndrome. III. Effects of emotions. Gastroenterology, 40, 27-36.

Connell, A. M., and Logan, C. J. H. (1967). The role of gastrin in the gastro-ileo-colic responses. American Journal of Digestive Diseases, 12, 277-284.

Connell, A. M., and Rowlands, E. N. (1960). Wireless telemetering from the digestive tract. Gut, 1, 266-272.

Cummings, J. H. (1973). Dietary fibre. Gut, 14, 69-81.

Davignon, J., Simmonds, W. J., and Ahrens, E. H. (1968). Usefulness of chromic oxide as an internal standard for balance studies in formula-fed patients and for assessment of colonic function. Journal of Clinical Investigation, 47, 127-138.

Eastwood, M. A., Kirkpatrick, J. R., Mitchell, W. D., Bone, A., and Hamilton, T. (1973). Effects of dietary supplements of wheat bran and cellulose on faeces and bowel functions. British Medical Journal, 4, 392-394.

Edwards, D. A. W., and Beck, E. R. (1971). Fecal flow, mixing and consistency. American Journal of Digestive Diseases, 16, 706-708.

Eve, I. S. (1966). A review of the physiology of the gastrointestinal tract in relation to radiation doses from radioactive materials. Health Physics, 12, 131-161.

Findlay, J. M., Mitchell, W. D., Eastwood, M. A., Anderson, A. J. B., and Smith, A. N. (1974). Intestinal streaming 
patterns in cholerrhoeic enteropathy and diverticular disease. Gut, 15, 207-212.

Hansky, J., and Connell, A. M. (1962). Measurement of gastrointestinal transit using radioactive chromium. Gut, $3,187-188$.

Hertz, A. F., Morton, C. J., Cook, F., Cox, A. N., Gardiner, H., Schlesinger, E. G., and Todd, A. H. (1907). The passage of food along the human alimentary canal. Guy's Hospital Reports, 61, 389-427.

Hinton, J. M., Lennard-Jones, J. E., and Young, A. C. (1969). A new method for studying gut transit times using radioopaque markers. Gut, 10, 842-847.

Hoelzel, F. (1930). The rate of passage of inert materials through the digestive tract. American Journal of Physiology, 92, 466-497.

Holdstock, D. J., Misiewicz, J. J., Smith, T., and Rowlands, E. N. (1970). Propulsion (mass movements) in the human colon and its relationship to meals and somatic activity. Gut, 11, 91-99.

Holdstock, D. J., and Misiewicz, J. J. (1970). Factors controlling colonic motility: colonic pressures and transit after meals in patients with total gastrectomy, pernicious anaemia or duodenal ulcer. Gut, 11, 100-110.

Jenkins, D. J. A., Hill, M. J. and Cummings, J. H. (1975). The effect of wheat fibre on blood lipids, faecal steroid excretion and serum iron. American Jourral of Clinical Nutrition, 28, 1408-1411.

Kirwan, W. O., and Smith, A. N. (1974). Gastrointestinal transit estimated by an isotope capsule. Scandinavian Journal of Gastroenterology, 9, 763-766.

Levitt, M. D., and Duane, W. C. (1972). Floating stoolsflatus versus fat. New England Journal of Medicine, 286, 973-975.

Mulinos, M. G. (1935). The value of selective drugs in the treatment of constipation. Review of Gastroenterology, 2, 292-301.

Payler, D. K., Pomare, E. W., Heaton, K. W., and Harvey, R. F. (1975). The effect of wheat bran on intestinal transit. Gut, 16, 209-213.

Scheline, R. R. (1973). Metabolism of foreign compounds by gastrointestinal microorganisms. Pharmacological Reviews, 25, 451-523.

Southgate, D. A. T. (1969). Determination of carbohydrates in food. 2. Unavailable carbohydrates. Journal of the Science of Food and Agriculture, 20, 331-335.

Southgate, D. A. T. (1973). Fibre and the other unavailable carbohydrates and their effects on the energy value of the diet. Proceedings of the Nutrition Society, 32, 131-136.

Wallace, R. P., Ehrenfeld, I., Cowett, M. P., Joliffe, N., Shapiro, L. L., and Sturtevant, M. (1938). Motility of the gastrointestinal tract. American Journal of Roentgenology, 39, 64-66.

West, R. C. (1973-74). Handbook of Chemistry and Physics, 54th edn. C.R.C. Press: Cleveland, Ohio.

Zilversmit, D. B. (1960). The design and analysis of isotope experiments. American Journal of Medicine, 29, 832-848. 